Marquette University

e-Publications@Marquette

\title{
The Crystal and Molecular Structure of a Trifluoroacetylacetonate Complex of Scandium, $\mathrm{Sc}\left(\mathrm{CH}_{3} \mathrm{COCHCOCF}_{3}\right)_{3}$
}

\author{
Dennis W. Bennett \\ University of Wisconsin - Milwaukee \\ Tasneem Siddiquee \\ University of Wisconsin - Milwaukee \\ Daniel T. Haworth \\ Marquette University \\ Sergey V. Lindeman \\ Marquette University, sergey.lindeman@marquette.edu
}

Follow this and additional works at: https://epublications.marquette.edu/chem_fac

Part of the Chemistry Commons

\section{Recommended Citation}

Bennett, Dennis W.; Siddiquee, Tasneem; Haworth, Daniel T.; and Lindeman, Sergey V., "The Crystal and Molecular Structure of a Trifluoroacetylacetonate Complex of Scandium, $\mathrm{Sc}\left(\mathrm{CH}_{3} \mathrm{COCHCOCF}_{3}\right)_{3}$ " (2007). Chemistry Faculty Research and Publications. 638.

https://epublications.marquette.edu/chem_fac/638 


\section{Marquette University \\ e-Publications@Marquette}

\section{Chemistry Faculty Research and Publications/College of Arts and Science}

This paper is NOT THE PUBLISHED VERSION; but the author's final, peer-reviewed manuscript. The published version may be accessed by following the link in the citation below.

Journal of Chemical Crystallography, Vol. 37, No. 3 (March 2007): 207-212. DOI. This article is (C) Springer Science+Business Media, LLC and permission has been granted for this version to appear in e-Publications@Marquette. Springer Science+Business Media, LLC does not grant permission for this article to be further copied/distributed or hosted elsewhere without the express permission from Springer Science+Business Media, LLC.

\section{The crystal and molecular structure of a trifluoroacetylacetonate complex of scandium, $\mathrm{Sc}\left(\mathrm{CH}_{3} \mathrm{COCHCOCF}\right)_{3}$}

Dennis W. Bennett

Department of Chemistry, University of Wisconsin - Milwaukee, Milwaukee, WI

Tasneem A. Siddiquee

Department of Chemistry, University of Wisconsin - Milwaukee, Milwaukee, WI Daniel T. Haworth

Department of Chemistry, Marquette University, Milwaukee, WI

Department of Chemistry, University of Wisconsin - Milwaukee, Milwaukee, WI Sergey $\mathrm{V}$. Lindeman

Department of Chemistry, Marquette University, Milwaukee, WI

The crystal and molecular structure of $\mathrm{Sc}\left(\mathrm{CH}_{3} \mathrm{COCHCOCF}_{3}\right)_{3}$ has been determined by X-ray diffraction. The compound crystallizes as pure mer-isomer in the orthorhombic space 
group Pbca with lattice parameters $a=15.166(8) \AA, b=13.560(7) \AA, c=19.327(10)$ $\AA, \alpha=\beta=\gamma=90^{\circ}, V=3974(4) \AA^{3}, Z=8$. The complex at $100 \mathrm{~K}$ is partially disordered in the crystal structure in an approximate $5: 1$ ratio with $83 \%$ fluorine population at C-11 and $17 \%$ at C-15. NMR data is compared to that previously reported.

\section{KEY WORDS}

Trifluoroacetylactonate, scandium acac, dipole moment

\section{Introduction}

We have previously reported on the syntheses and characterization of nine scandium (III) $\beta$ diketonate complexes which have a fluorosubstituent. ${ }^{1}$ Prior to this report only three other fluorinated $\beta$-diketonates had been reported. ${ }^{2,3}$ The dipole moment of six of these compounds, $\mathrm{Sc}(\mathrm{RCOCHCOR})_{3}$ increases with the fluorine content of the $\mathrm{R}^{\prime}$ group from 4.34 to $6.18 \mathrm{D}$. A variable ${ }^{19} \mathrm{~F} \mathrm{NMR}$ study of all compounds gave one symmetric resonance in the range of $-56^{\circ} \mathrm{C}$ to $-65^{\circ} \mathrm{C}$. Since stereochemical non-rigidity has been cited as a characteristic property of six coordinate scandium complexes it was likely that these complexes were fluxional. ${ }^{4}$ Holm has shown that because the methyl resonance of the $\mathrm{Sc}(\mathrm{pmhd})_{3}$ (pmhd=1-phenyl-5-methylhexane-1,3-dionate) could not be made enantiotopic at $-95^{\circ} \mathrm{C}$ a low rearrangement barrier was indicated; whereas, the corresponding $\mathrm{Al}(\mathrm{pmhd})_{3}$ and $\mathrm{Ga}(\mathrm{pmhd})_{3}$ complexes were non-rigid. ${ }^{4} \mathrm{An}$ X-ray structural determination was undertaken of $\mathrm{Sc}\left(\mathrm{CH}_{3} \mathrm{COCHCOCF}_{3}\right)_{3}$ to determine its conformation in the solid state and to reevaluate its ${ }^{19} \mathrm{~F}$ NMR. A comparison to the known X-ray structure of $\mathrm{Sc}\left(\mathrm{CH}_{3} \mathrm{COCHCOCH}\right)_{3}$ will also be carried out. ${ }^{5}$

Table 1. Crystal Data and Structure Refinement for Sc(tfac) ${ }_{3}$

CCDC deposition number

Empirical formula

Formula weight

Temperature

Wavelength

Crystal system

Space group

Unit cell dimensions

\begin{tabular}{|ll|}
\hline$a(\AA)$ & $15.166(8)$ \\
\hline$b(\AA)$ & $13.560(7)$ \\
\hline$c(\AA)$ & $19.327(10)$ \\
\hline$a\left(^{\circ}\right)$ & 90 \\
\hline$\beta\left(^{\circ}\right)$ & 90 \\
\hline$\gamma\left(^{\circ}\right)$ & 90 \\
\hline Volume $\left(\AA^{3}\right)$ & $3974(4)$ \\
\hline
\end{tabular}

\section{3}

$\mathrm{C}_{15} \mathrm{H}_{12} \mathrm{~F}_{9} \mathrm{O}_{6} \mathrm{Sc}$

504.21

100(2) K

$0.71073 \AA$

Orthorhombic

Pbca 


\begin{tabular}{|ll|}
\hline$Z$ & 8 \\
\hline Density (calculated) & $1.685 \mathrm{Mg} / \mathrm{m}^{3}$ \\
\hline Absorption coefficient & $0.483 \mathrm{~mm}^{-1}$ \\
\hline F(000) & 2016 \\
\hline Crystal size & $0.22 \times 0.14 \times 0.07 \mathrm{~mm}^{3}$ \\
\hline Theta range for data collection & $2.11-31.96^{\circ}$. \\
\hline Index ranges & $0 \leq h \leq 21$ \\
\hline & $0 \leq k \leq 19$ \\
\hline & $0 \leq I \leq 27$ \\
\hline Reflections collected & 64660 \\
\hline Independent reflections & $6593[R$ int $=0.0543]$ \\
\hline Completeness to theta=31.96 & $95.7 \%$ \\
\hline Absorption correction & Semi-empirical from equivalents \\
\hline Max. and min. transmission & 0.9670 and 0.9012 \\
\hline Refinement method & Full-matrix least-squares on $F^{2}$ \\
\hline Data/restraints/parameters & $6593 / 6 / 329$ \\
\hline Goodness-of-fit on $F^{2}$ & 1.020 \\
\hline Final $R$ indices [I $>2 \sigma(I)]$ & $R{ }_{1}=0.0562, W R{ }_{2}=0.1437$ \\
\hline$R$ indices (all data) & $R{ }_{1}=0.0928, W R{ }_{2}=0.1617$ \\
\hline Largest diff. peak and hole & 0.600 and $-0.399\left(e \AA^{-3}\right)$ \\
\hline
\end{tabular}

\section{Experimental}

The crystals of the title compound were obtained by recrystallization from ethanol-methylene chloride. A crystal $\left(0.22 \times 0.14 \times 0.07 \mathrm{~mm}^{3}\right)$ was attached to a glass fiber and mounted on a Bruker APEX Z CCD diffractometer. The data were collected at $100 \mathrm{~K}$ using graphite monochromatized MoKaradiation $(\lambda=0.71073 \AA)$ and the $\theta / 2 \theta$ mode in the $\theta$ range 2.11$31.96^{\circ}$. An absorption correction was made from semi-empirical equivalents. The structure was solved by direct methods and refined by full-matrix least squares based on $F^{2.6} \mathrm{~A}$ total of 64660 reflections were collected (6593 independent reflections, $R$ int $=0.0543$ ). The final refinement resulted in $R=0.0562$ and $w R_{2}=0.1437$. All hydrogen atoms were located in a series of difference Fourier syntheses and were refined isotropically, except for methyl groups, where the geometric constraints on hydrogens were put to avoid the negative effects on refinement of the corresponding fluorines. The experimental data are given in Table 1 and the structural parameters are given in Tables 2-5.

Table 2. Atomic Coordinates $\left(\times 10^{4}\right)$ and Equivalent Isotropic Displacement Parameters $\left(\AA^{2} \times 10^{3}\right)$ for $\mathrm{Sc}(\mathrm{tfac})_{3}$

\begin{tabular}{|lllll|}
\hline & $\boldsymbol{x}$ & $\boldsymbol{y}$ & $\boldsymbol{z}$ & $\boldsymbol{U}(\mathbf{e q})$ \\
\hline $\operatorname{Sc}(1)$ & $1343(1)$ & $7543(1)$ & $2385(1)$ & $27(1)$ \\
\hline
\end{tabular}




\begin{tabular}{|c|c|c|c|c|}
\hline & $x$ & $y$ & $z$ & $U(e q)$ \\
\hline $\mathrm{O}(1)$ & $2057(1)$ & $6792(1)$ & $3138(1)$ & $32(1)$ \\
\hline $\mathrm{O}(2)$ & 2386(1) & 8543(1) & $2471(1)$ & $33(1)$ \\
\hline $\mathrm{O}(3)$ & $601(1)$ & $8355(1)$ & $3082(1)$ & $34(1)$ \\
\hline $\mathrm{O}(4)$ & $288(1)$ & $6562(1)$ & $2488(1)$ & $32(1)$ \\
\hline $\mathrm{O}(5)$ & $827(1)$ & $8244(1)$ & $1513(1)$ & $36(1)$ \\
\hline $\mathrm{O}(6)$ & $1930(1)$ & $6643(1)$ & $1622(1)$ & $35(1)$ \\
\hline$C(1)$ & $3081(2)$ & $6261(2)$ & $3974(1)$ & $46(1)$ \\
\hline$F(1)$ & $3008(1)$ & $5343(1)$ & $3750(1)$ & $63(1)$ \\
\hline$F(2)$ & 3918(1) & $6370(2)$ & 4164(1) & $73(1)$ \\
\hline$F(3)$ & $2588(2)$ & $6340(2)$ & $4531(1)$ & $88(1)$ \\
\hline$C(2)$ & 2788(1) & $7011(2)$ & $3426(1)$ & $33(1)$ \\
\hline$C(3)$ & $3302(2)$ & $7825(2)$ & $3313(1)$ & $41(1)$ \\
\hline$C(4)$ & $3066(1)$ & $8579(2)$ & 2842(1) & $34(1)$ \\
\hline$C(5)$ & $3645(2)$ & $9470(2)$ & $2781(2)$ & $46(1)$ \\
\hline$C(6)$ & $-344(2)$ & $8891(2)$ & $3961(1)$ & $45(1)$ \\
\hline$F(4)$ & $-364(1)$ & $9797(1)$ & $3709(1)$ & $53(1)$ \\
\hline$F(5)$ & $-1134(2)$ & $8725(2)$ & $4241(1)$ & $84(1)$ \\
\hline$F(6)$ & $237(2)$ & $8898(2)$ & $4470(1)$ & $84(1)$ \\
\hline$C(7)$ & $-97(2)$ & $8124(2)$ & $3415(1)$ & $33(1)$ \\
\hline$C(8)$ & $-611(2)$ & $7299(2)$ & $3331(1)$ & $38(1)$ \\
\hline$C(9)$ & $-396(1)$ & $6541(2)$ & $2858(1)$ & $33(1)$ \\
\hline$C(10)$ & $-988(2)$ & $5657(2)$ & 2793(2) & $41(1)$ \\
\hline$C(11)$ & $428(2)$ & $8769(2)$ & 392(1) & $48(1)$ \\
\hline$F(7)$ & $-398(1)$ & $8698(2)$ & $460(2)$ & $91(1)$ \\
\hline$F(8)$ & $694(2)$ & $9659(2)$ & $496(2)$ & $108(1)$ \\
\hline$F(9)$ & $588(2)$ & $8616(3)$ & $-275(1)$ & $104(1)$ \\
\hline$C(11)$ & $428(2)$ & $8769(2)$ & 392(1) & $48(1)$ \\
\hline$C(12)$ & $919(2)$ & $8056(2)$ & $873(1)$ & $39(1)$ \\
\hline$C(13)$ & 1396(2) & $7308(2)$ & $579(2)$ & $46(1)$ \\
\hline$C(14)$ & $1881(2)$ & $6626(2)$ & $973(1)$ & $38(1)$ \\
\hline$C(15)$ & $2388(2)$ & $5826(2)$ & $602(2)$ & $55(1)$ \\
\hline$F(7 A)$ & $2455(6)$ & $5095(6)$ & $1011(5)$ & $50(2)$ \\
\hline$F(8 A)$ & $3142(6)$ & $6230(7)$ & 496(5) & $59(3)$ \\
\hline$F(9 A)$ & $2009(7)$ & $5657(8)$ & $41(5)$ & $61(3)$ \\
\hline
\end{tabular}

Note. $U(\mathrm{eq})$ is defined as one third of the trace of the orthogonalized $U$ ittensor. 
The ${ }^{13} \mathrm{~F} \mathrm{NMR}$ was recorded in $\mathrm{CH}_{2} \mathrm{Cl}_{2}$, with $\mathrm{CFCl}_{3}$ as the internal standard on a GE 300-GN instrument operating at $282\left({ }^{19} \mathrm{~F}\right) \mathrm{MHZ}$.

Table 3. Bond Lengths $[\AA]]$ and Angles $\left[{ }^{\circ}\right]$ for Sc(tfac $)_{3}$

Bond lengths $(\AA)$

\begin{tabular}{|ll|}
\hline$S c(1)-O(3)$ & $2.0720(16)$ \\
\hline$S c(1)-O(1)$ & $2.0804(16)$ \\
\hline$S c(1)-O(5)$ & $2.0879(17)$ \\
\hline$S c(1)-O(4)$ & $2.0893(16)$ \\
\hline$S c(1)-O(2)$ & $2.0911(17)$ \\
\hline$S c(1)-O(6)$ & $2.1120(17)$ \\
\hline$O(1)-C(2)$ & $1.275(3)$ \\
\hline$O(2)-C(4)$ & $1.257(3)$ \\
\hline$O(3)-C(7)$ & $1.277(3)$ \\
\hline$O(4)-C(9)$ & $1.260(3)$ \\
\hline$O(5)-C(12)$ & $1.270(3)$ \\
\hline$O(6)-C(14)$ & $1.256(3)$ \\
\hline$C(1)-F(3)$ & $1.316(3)$ \\
\hline$C(1)-F(1)$ & $1.323(3)$ \\
\hline$C(1)-F(2)$ & $1.329(3)$ \\
\hline$C(1)-C(2)$ & $1.535(3)$ \\
\hline$C(2)-C(3)$ & $1.370(3)$ \\
\hline$C(3)-C(4)$ & $1.414(3)$ \\
\hline$C(4)-C(5)$ & $1.498(3)$ \\
\hline$C(6)-F(6)$ & $1.320(3)$ \\
\hline$C(6)-F(4)$ & $1.323(3)$ \\
\hline$C(6)-F(5)$ & $1.332(3)$ \\
\hline$C(6)-C(7)$ & $1.529(3)$ \\
\hline$C(7)-C(8)$ & $1.373(3)$ \\
\hline$C(8)-C(9)$ & $1.413(3)$ \\
\hline$C(9)-C(10)$ & $1.503(3)$ \\
\hline$C(11)-F(7)$ & $1.262(3)$ \\
\hline$C(11)-F(8)$ & $1.289(3)$ \\
\hline$C(11)-F(9)$ & $1.328(4)$ \\
\hline$C(11)-C(12)$ & $1.534(3)$ \\
\hline$C(12)-C(13)$ & $1.370(4)$ \\
\hline & \\
\hline & \\
\hline &
\end{tabular}




$\begin{array}{ll}C(13)-C(14) & 1.406(4) \\ C(14)-C(15) & 1.511(4)\end{array}$

Bond angles $\left({ }^{\circ}\right)$

\begin{tabular}{|ll}
\hline$O(3)-S c(1)-O(1)$ & $95.05(7)$ \\
\hline$O(3)-S c(1)-O(5)$ & $94.58(7)$ \\
\hline$O(1)-S c(1)-O(5)$ & $169.40(7)$ \\
\hline$O(3)-S c(1)-O(4)$ & $82.01(7)$ \\
\hline$O(1)-S c(1)-O(4)$ & $91.16(7)$ \\
\hline$O(5)-S c(1)-O(4)$ & $94.57(7)$ \\
\hline$O(3)-S c(1)-O(2)$ & $90.81(7)$ \\
\hline$O(1)-S c(1)-O(2)$ & $82.42(7)$ \\
\hline$O(5)-S c(1)-O(2)$ & $93.01(7)$ \\
\hline$O(4)-S c(1)-O(2)$ & $169.94(7)$ \\
\hline$O(3)-S c(1)-O(6)$ & $172.08(6)$ \\
\hline$O(1)-S c(1)-O(6)$ & $89.21(7)$ \\
\hline$O(5)-S c(1)-O(6)$ & $81.77(7)$ \\
\hline$O(4)-S c(1)-O(6)$ & $91.25(7)$ \\
\hline$O(2)-S c(1)-O(6)$ & $96.38(7)$ \\
\hline$C(2)-O(1)-S c(1)$ & $130.02(14)$ \\
\hline$C(4)-O(2)-S c(1)$ & $133.73(14)$ \\
\hline$C(7)-O(3)-S c(1)$ & $130.28(14)$ \\
\hline$C(9)-O(4)-S c(1)$ & $134.30(14)$ \\
\hline$C(12)-O(5)-S c(1)$ & $130.83(15)$ \\
\hline$C(14)-O(6)-S c(1)$ & $133.03(15)$ \\
\hline$F(3)-C(1)-F(1)$ & $107.3(2)$ \\
\hline$F(3)-C(1)-F(2)$ & $107.9(2)$ \\
\hline$F(1)-C(1)-F(2)$ & $106.0(2)$ \\
\hline$F(3)-C(1)-C(2)$ & $110.2(2)$ \\
\hline$F(1)-C(1)-C(2)$ & $111.9(2)$ \\
\hline$F(2)-C(1)-C(2)$ & $113.2(2)$ \\
\hline$O(1)-C(2)-C(3)$ & $127.9(2)$ \\
\hline$O(1)-C(2)-C(1)$ & $113.49(19)$ \\
\hline$C(3)-C(2)-C(1)$ & $118.6(2)$ \\
\hline$C(2)-C(3)-C(4)$ & $122.7(2)$ \\
\hline$O(2)-C(4)-C(3)$ & $123.2(2)$ \\
\hline$O(2)-C(4)-C(5)$ & $117.8(2)$ \\
\hline$O$ & \\
\hline$O$ &
\end{tabular}




\begin{tabular}{|c|c|}
\hline$C(3)-C(4)-C(5)$ & $119.0(2)$ \\
\hline$F(6)-C(6)-F(4)$ & $106.4(2)$ \\
\hline$F(6)-C(6)-F(5)$ & $107.5(2)$ \\
\hline$F(4)-C(6)-F(5)$ & $106.6(2)$ \\
\hline$F(6)-C(6)-C(7)$ & $110.8(2)$ \\
\hline$F(4)-C(6)-C(7)$ & $112.4(2)$ \\
\hline$F(5)-C(6)-C(7)$ & $112.7(2)$ \\
\hline $\mathrm{O}(3)-\mathrm{C}(7)-\mathrm{C}(8)$ & $127.7(2)$ \\
\hline $\mathrm{O}(3)-\mathrm{C}(7)-\mathrm{C}(6)$ & $112.6(2)$ \\
\hline$C(8)-C(7)-C(6)$ & $119.7(2)$ \\
\hline$C(7)-C(8)-C(9)$ & $122.5(2)$ \\
\hline $\mathrm{O}(4)-\mathrm{C}(9)-\mathrm{C}(8)$ & $122.7(2)$ \\
\hline $\mathrm{O}(4)-\mathrm{C}(9)-\mathrm{C}(10)$ & $117.5(2)$ \\
\hline$C(8)-C(9)-C(10)$ & $119.8(2)$ \\
\hline$F(7)-C(11)-F(8)$ & $111.4(3)$ \\
\hline$F(7)-C(11)-F(9)$ & $105.7(3)$ \\
\hline$F(8)-C(11)-F(9)$ & 103.9(3) \\
\hline$F(7)-C(11)-C(12)$ & $111.7(2)$ \\
\hline$F(8)-C(11)-C(12)$ & $110.1(2)$ \\
\hline$F(9)-C(11)-C(12)$ & 113.6(3) \\
\hline $\mathrm{O}(5)-\mathrm{C}(12)-\mathrm{C}(13)$ & $127.7(2)$ \\
\hline $\mathrm{O}(5)-\mathrm{C}(12)-\mathrm{C}(11)$ & $114.2(2)$ \\
\hline$C(13)-C(12)-C(11)$ & $118.1(2)$ \\
\hline$C(12)-C(13)-C(14)$ & $122.5(3)$ \\
\hline $\mathrm{O}(6)-\mathrm{C}(14)-\mathrm{C}(13)$ & $124.1(2)$ \\
\hline $\mathrm{O}(6)-\mathrm{C}(14)-\mathrm{C}(15)$ & $117.1(2)$ \\
\hline$C(13)-C(14)-C(15)$ & $118.7(2)$ \\
\hline
\end{tabular}

Note. Symmetry transformations used to generate equivalent atoms.

\section{Results and discussion}

A drawing of the mer-molecule and its crystallographic numbering is given in Fig. 2.

The title compound gave a structure which is partially disordered in the crystal due to the mer- isomer of $\mathrm{Sc}\left(\mathrm{CH}_{3} \mathrm{COCHCOCF}_{3}\right)_{3}$ having an approximate population of the $\mathrm{CF}_{3}$ groups in a ratio of $5: 1$ with $83 \%$ population at C-11 and $17 \%$ at C-15 (Fig. 1). These are related by $180^{\circ}$ rotation around a quasi-two-fold axis going through $\mathrm{C}(13), \mathrm{Sc}(1)$ and the bisectral plane between $\mathrm{O}(1)$ and $\mathrm{O}(3)$ (Fig. 3). 
Table 4. Anisotropic Displacement Parameters $\left(\AA^{2} \times 10^{3}\right)$ for Sc(tfac $)_{3}$

\begin{tabular}{|c|c|c|c|c|c|c|}
\hline & $U^{11}$ & $U^{22}$ & $U^{33}$ & $U^{23}$ & $U^{13}$ & $U^{12}$ \\
\hline $\operatorname{Sc}(1)$ & $23(1)$ & $23(1)$ & $34(1)$ & $2(1)$ & $0(1)$ & $2(1)$ \\
\hline $\mathrm{O}(1)$ & $32(1)$ & $27(1)$ & $38(1)$ & $6(1)$ & $-3(1)$ & $-1(1)$ \\
\hline $\mathrm{O}(2)$ & $28(1)$ & $26(1)$ & $44(1)$ & $3(1)$ & $2(1)$ & $-1(1)$ \\
\hline $\mathrm{O}(3)$ & $31(1)$ & $28(1)$ & $42(1)$ & $-3(1)$ & $3(1)$ & 2(1) \\
\hline $\mathrm{O}(4)$ & $26(1)$ & $26(1)$ & $45(1)$ & $-1(1)$ & $1(1)$ & $1(1)$ \\
\hline $\mathrm{O}(5)$ & $38(1)$ & $31(1)$ & $39(1)$ & $5(1)$ & $-5(1)$ & $4(1)$ \\
\hline $\mathrm{O}(6)$ & $31(1)$ & $34(1)$ & $40(1)$ & $-3(1)$ & $2(1)$ & $5(1)$ \\
\hline$C(1)$ & $52(2)$ & $46(1)$ & $40(1)$ & $3(1)$ & $-13(1)$ & $5(1)$ \\
\hline$F(1)$ & $85(1)$ & $39(1)$ & $66(1)$ & $11(1)$ & $-26(1)$ & $9(1)$ \\
\hline$F(2)$ & $63(1)$ & $74(1)$ & $81(1)$ & $14(1)$ & $-39(1)$ & $3(1)$ \\
\hline$F(3)$ & $114(2)$ & $101(2)$ & $48(1)$ & $26(1)$ & $19(1)$ & $47(1)$ \\
\hline$C(2)$ & $34(1)$ & $30(1)$ & $33(1)$ & $-2(1)$ & $-4(1)$ & $4(1)$ \\
\hline$C(3)$ & $35(1)$ & $36(1)$ & $50(1)$ & $-4(1)$ & $-12(1)$ & $-2(1)$ \\
\hline$C(4)$ & $26(1)$ & $27(1)$ & $51(1)$ & $-8(1)$ & $7(1)$ & $-1(1)$ \\
\hline$C(5)$ & $32(1)$ & $29(1)$ & $78(2)$ & $-7(1)$ & $5(1)$ & $-5(1)$ \\
\hline$C(6)$ & $55(2)$ & $41(1)$ & $38(1)$ & $-3(1)$ & $11(1)$ & $8(1)$ \\
\hline$F(4)$ & $72(1)$ & $34(1)$ & $53(1)$ & $-5(1)$ & $10(1)$ & $15(1)$ \\
\hline$F(5)$ & $96(2)$ & $68(1)$ & 89(1) & $-18(1)$ & $60(1)$ & $-3(1)$ \\
\hline$F(6)$ & $125(2)$ & $77(1)$ & $51(1)$ & $-24(1)$ & $-31(1)$ & $44(1)$ \\
\hline$C(7)$ & $36(1)$ & $29(1)$ & $34(1)$ & $3(1)$ & $1(1)$ & $10(1)$ \\
\hline$C(8)$ & $32(1)$ & $33(1)$ & 48(1) & $6(1)$ & $11(1)$ & $3(1)$ \\
\hline$C(9)$ & $25(1)$ & $26(1)$ & $47(1)$ & $7(1)$ & $-4(1)$ & $3(1)$ \\
\hline$C(10)$ & $29(1)$ & $30(1)$ & $65(2)$ & $6(1)$ & $1(1)$ & $-2(1)$ \\
\hline$C(11)$ & $46(1)$ & $52(2)$ & $44(1)$ & $14(1)$ & $-15(1)$ & $-12(1)$ \\
\hline$F(7)$ & $38(1)$ & $120(2)$ & $116(2)$ & $81(2)$ & $-7(1)$ & $2(1)$ \\
\hline$F(8)$ & $153(3)$ & $49(1)$ & $122(2)$ & $41(1)$ & $-97(2)$ & $-36(2)$ \\
\hline$F(9)$ & $111(2)$ & $159(3)$ & $43(1)$ & $28(2)$ & $-14(1)$ & $42(2)$ \\
\hline$C(11)$ & $46(1)$ & $52(2)$ & $44(1)$ & $14(1)$ & $-15(1)$ & $-12(1)$ \\
\hline$C(12)$ & $36(1)$ & $39(1)$ & $40(1)$ & $8(1)$ & $-9(1)$ & $-11(1)$ \\
\hline$C(13)$ & $47(1)$ & $57(2)$ & $36(1)$ & $-5(1)$ & $-7(1)$ & $-5(1)$ \\
\hline$C(14)$ & $30(1)$ & $41(1)$ & $44(1)$ & $-8(1)$ & $2(1)$ & $-7(1)$ \\
\hline$C(15)$ & $45(2)$ & $63(2)$ & $57(2)$ & $-23(1)$ & $4(1)$ & $1(1)$ \\
\hline
\end{tabular}

Note. The anisotropic displacement factor exponent takes the form: $-2 \pi^{2}\left[h^{2} a^{*} U^{11}+\cdots+2 h k\right.$ $\left.a{ }^{*} b^{*} \mathrm{U}^{12}\right]$. 
Our previous studies had shown that the compound has a large dipole moment (4.34 D) and only one ${ }^{19} \mathrm{~F}$ NMR was observed in the range

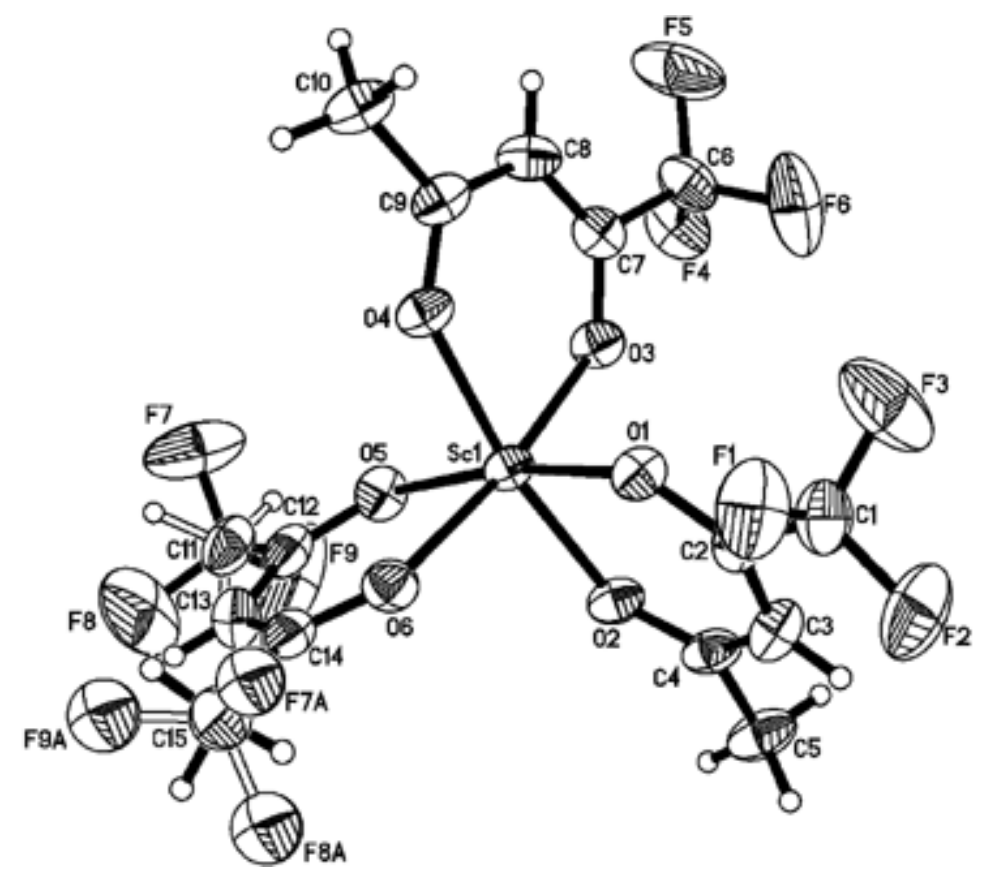

Fig. 1. ORTEP diagram showing both $\mathrm{CF}_{3}$ contributions with $\mathrm{C}(11)$ having $83 \%$.

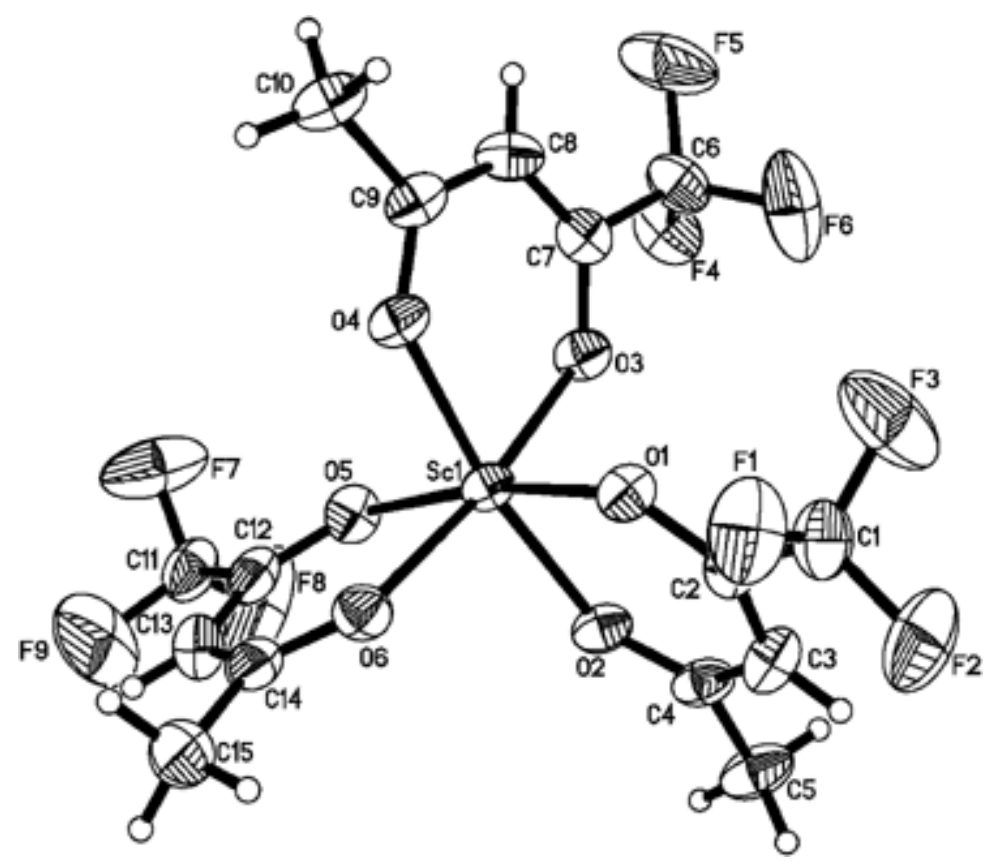

Fig. 2. The molecular structure of mer-Sc $\left(\mathrm{CH}_{3} \mathrm{COCHCF}_{3}\right)_{3}$. Ellipsoids are shown at the $50 \%$ probability level. 


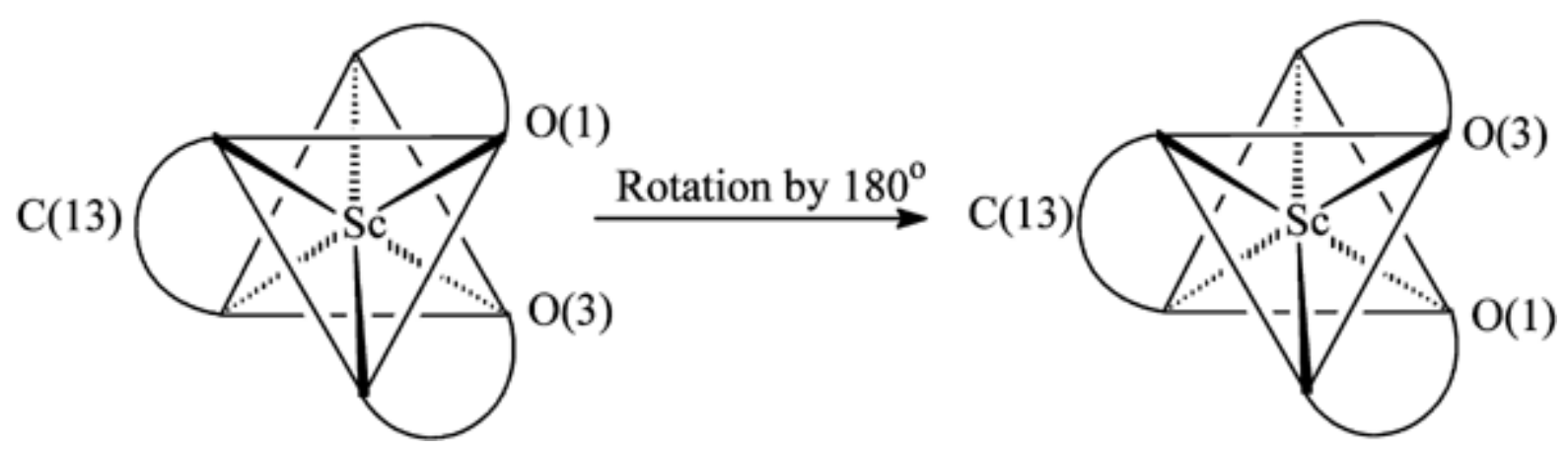

Fig. 3. Relation between the different $\mathrm{CF}_{3}$ populations in the disordered structure.

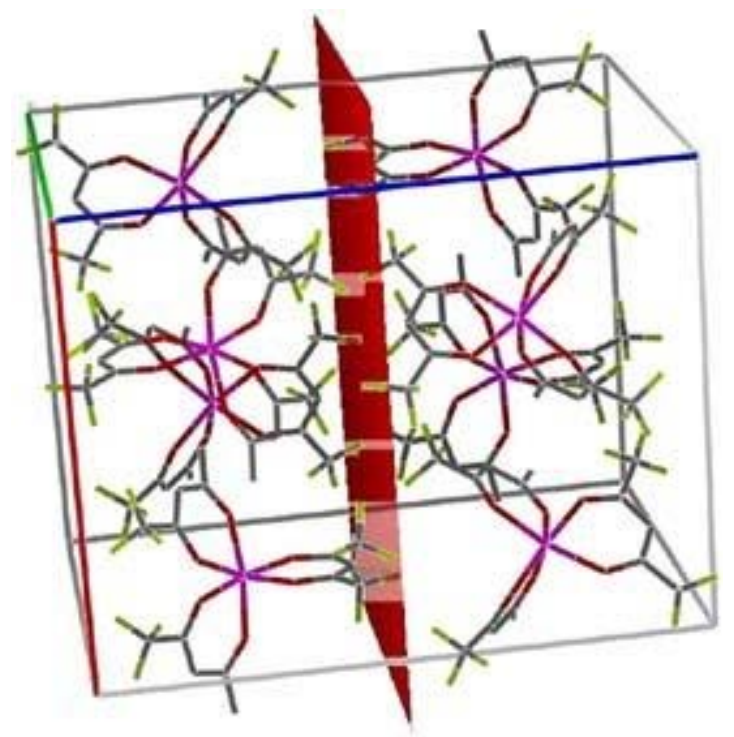

Fig. 4. Packing diagram of mer-Sc($\left(\mathrm{CH}_{3} \mathrm{COCHCF}_{3}\right)_{3}$ along $b$-axis.

of $-56^{\circ}$ to $-65^{\circ} \mathrm{C}$. While this data alone cannot be used to support stereochemical nonrigidity, a reexamination of its ${ }^{19} \mathrm{~F}$ NMR shows only one $\mathrm{CF}_{3}$ resonance at room temperature (-76.2 ppm).

Table 3 shows that the $\mathrm{CF}_{3}$ group lengthens the $\mathrm{C}-\mathrm{O}$ bond and shortens the $\mathrm{Sc}-\mathrm{O}$ bond adjacent to the $\mathrm{CF}_{3}$ group when compared to the corresponding bonds adjacent to the $\mathrm{CH}_{3}$ groups. The acidic character of the hydrogens at $\mathrm{C}(3), \mathrm{C}(8)$ and $\mathrm{C}(13)$ is in agreement with the shortening of the $\mathrm{C}-\mathrm{H}$ bonds due to the shift of electron density toward these carbon atoms. The influence of the $\mathrm{CF}_{3}$ group on the shorter $\mathrm{Sc}-\mathrm{O}$ and larger $\mathrm{O}-\mathrm{C}$ bond lengths is shown in Table 3. The Sc-O bond distances are all smaller than the $2.20 \AA$ predicted on the basis of $0.81 \AA$ for scandium ion using the Pauling scale. The title compound minus the

Table 5. Hydrogen coordinates $\left(\times 10^{4}\right)$ and isotropic displacement parameters $\left(\AA^{2} \times 10^{3}\right)$ for $\operatorname{Sc}(\operatorname{tfac})_{3}$

\begin{tabular}{|lllll|}
\hline & $\boldsymbol{x}$ & $\boldsymbol{y}$ & $\boldsymbol{z}$ & $\boldsymbol{U}(\mathbf{e q})$ \\
\hline $\mathrm{H}(15 \mathrm{~A})$ & 129 & 9273 & 669 & 71 \\
\hline $\mathrm{H}(15 \mathrm{~B})$ & -9 & 8403 & 121 & 71 \\
\hline $\mathrm{H}(15 \mathrm{C})$ & 850 & 9086 & 79 & 71 \\
\hline
\end{tabular}




\begin{tabular}{|lllll|}
\hline & $\boldsymbol{x}$ & $\boldsymbol{y}$ & $\boldsymbol{z}$ & $\boldsymbol{U}(\mathbf{e q})$ \\
\hline$H(15 \mathrm{D})$ & 2868 & 5591 & 899 & 83 \\
\hline $\mathrm{H}(15 \mathrm{E})$ & 2635 & 6091 & 172 & 83 \\
\hline $\mathrm{H}(15 \mathrm{~F})$ & 1991 & 5277 & 495 & 83 \\
\hline$H(3)$ & $3810(20)$ & $7910(20)$ & $3507(16)$ & $55(8)$ \\
\hline$H(5 \mathrm{~A})$ & $4220(30)$ & $9240(30)$ & $2783(19)$ & $78(11)$ \\
\hline$H(5 B)$ & $3520(20)$ & $9870(30)$ & $3160(20)$ & $80(11)$ \\
\hline$H(5 \mathrm{C})$ & $3570(20)$ & $9860(20)$ & $2364(17)$ & $56(9)$ \\
\hline$H(8)$ & $-1110(20)$ & $7260(20)$ & $3552(16)$ & $51(8)$ \\
\hline$H(10 A)$ & $-1610(20)$ & $5880(20)$ & $2829(15)$ & $48(8)$ \\
\hline$H(10 B)$ & $-930(20)$ & $5300(20)$ & $2382(16)$ & $49(8)$ \\
\hline$H(10 \mathrm{C})$ & $-840(20)$ & $5250(20)$ & $3143(17)$ & $57(9)$ \\
\hline$H(13)$ & $1450(20)$ & $7200(30)$ & $166(19)$ & $67(11)$ \\
\hline
\end{tabular}

$\mathrm{CF}_{3}$ and $\mathrm{CH}_{3}$ groups adopts a distorted octahedron $\left(\mathrm{D}_{3}\right)$ structure as indicated by the two types of O-O separation of the order of 2.742(11) and 3.046(55) $\AA$ observed in the complex. The twist angles between fac-triangle $\mathrm{O}(1) \mathrm{O}(4) \mathrm{O}(6)$ and $\mathrm{O}(2) \mathrm{O}(3) \mathrm{O}(5)$ is about $48.2^{\circ}$ which is less than $60^{\circ}$ required for an ideal octahedral geometry. The dihedral angles between the chelate rings are, as a result, somewhat smaller $\left(84.7(1)^{\circ}\right)$ than the ideal value, $90^{\circ}$. The data is similar to that reported for $\mathrm{Sc}(\mathrm{acac}) 3 .{ }^{5}$ The packing diagram (Fig. 4) reveals that the $\mathrm{CF}_{3}$ groups tend to occupy the space in a plane, on an average parallel to the $a b$-plane.

The IR spectrum shows the characteristic carbonyl stretching frequency at $1618 \mathrm{~cm}^{-1}$.

X-ray diffraction powder data on mer-Sc(tfac) 3 have been reported and the indexing of the diffraction patterns were carried out using crystal data for mer-Fe(tfac) ${ }_{3}{ }^{7,8}$ The data indicates that these compounds are isostructural with unit cell parameters and values in the range of data shown in Table 1.

The data taken as a whole would indicate that $\mathrm{Sc}(\operatorname{tfac})_{3}$ is nonrigid in solution.

\section{Notes}

\section{Acknowledgement}

DTH acknowledges M. Das for assistance with the synthesis of the title compound, to Marquette University for the purchase of the diffractometer used in this study, and T. Zeczycki for assistance in recording the NMR.

\section{Supplementary material}

CCDC 298953 contains the supplementary crystallographic data and can be obtained free of charge at www.ccdc.cam.ac.uk/conts/retrieving.html or from the Cambridge Crystallographic 
Data Centre (CCDC), 12 Union Road, Cambridge CB2 1EZ, UK; fax: +44(0)1223-336033; email: deposit@ccdc.cam.ac.uk.

\section{References}

${ }^{1}$ Haworth, D. T.; Das, M. Synth. React. Inorg. Met-Org. Chem. 1984, 14, 283.

${ }^{2}$ Sievers, R. E.; Connelly, J. W. Inorg. Synth. 1971, 12, 72.

${ }^{3}$ Berg, E. W.; Costa, J. J. C. Anal. Chim. Acta. 1968, 40, 101.

${ }^{4}$ Hutchinson, J. R.; Gordon, J. C.; Holm, R. H. Inorg. Chem. 1971, 10, 1004.

${ }^{5}$ Anderson, T. J.; Neuman, M. A.; Melson, G. A. Inorg. Chem. 1973, 12, 927.

${ }^{6}$ Sheldrick, G. M. SHELXL-97, Software for Crystal Structure Determination, Institut für Anorganishche Chemie der Univesität Tammannstra, 4, 37077 Göttingen, Germany.

7Lisoivan, V. I.; Gromilov, S. A. Russian J. Inorg. Chem. 1988, 33, 1482.

${ }^{8}$ Gromilov, S. A.; Lisoivan, V. I.; Baidina, I. A.; Borisov, S. V. Zhuk. Struk. Khim. 1990, 31, 127. 